\title{
Stability analysis of a class of two-dimensional multipolar vortex equilibria
}

\author{
Darren Crowdy and Martin Cloke ${ }^{\text {b) }}$ \\ Department of Mathematics, Imperial College of Science, Technology and Medicine, 180 Queen's Gate, \\ London SW7 2BZ, United Kingdom
}

(Received 12 June 2001; accepted 14 March 2002; published 2 May 2002)

\begin{abstract}
The stability properties of a class of explicit multipolar vortex solutions of the two-dimensional Euler equations found in Crowdy [Phys. Fluids. 11, 2556 (1999)] are studied. While the tripole solutions are linearly unstable in all configurations, it is found that the exact quadrupolar vortices have distinguished linear stability properties revealing them to be neutrally stable in all configurations. This result is consistent with observations by previous investigators on the general robustness of quadrupolar vortex structures. Higher-order multipolar structures are linearly unstable when the satellites are too close together, but become neutrally stable when the satellites are far enough apart and the ambient vortex patch sufficiently distorted. The nonlinear evolution of perturbed solutions is investigated numerically using contour dynamics methods. Some new results concerning limiting states involving cusp singularities in the vortex patch boundaries are also presented. (C) 2002 American Institute of Physics. [DOI: 10.1063/1.1476302]
\end{abstract}

\section{INTRODUCTION}

Multipolar vortices are a recurring dynamical feature in many large-scale geophysical, atmospheric, and astrophysical flows. The term refers to a collection of coherent vortex structures with distributions of vorticity having certain characteristic geometrical arrangements. Monopoles, dipoles, tripoles, and quadrupoles, for example, refer to vorticity distributions having one, two, three, and four distinct vorticity extrema. Higher order structures have correspondingly larger numbers of such extrema. Typically, in an $n$-polar vortex a central core of vorticity of one sign is shielded by $n-1$ satellite vortices all of opposite sign such that the total circulation of the structure is approximately zero and the combined structure rotates steadily. One way in which multipolar vortices have been observed to form is via the instability of shielded monopolar structures. A linear stability analysis of a shielded two-contour Rankine vortex by Flierl ${ }^{2}$ showed the possibility of the formation of higher-order multipolar structures from the barotropic instability of shielded monopoles.

Once formed, the stability of these multipolar vortices is of great interest and has attracted the efforts of many researchers. The monopole and dipole have long been studied in the literature. The form and structure of the tripole was studied in detail by Polvani and Carton ${ }^{3}$ and most instances shown to be stable. The case of the quadrupole has also received much attention in the last decade, not least because it appears to be relatively robust compared to higher order structures. Carton ${ }^{4}$ observed the formation of (transient) quadrupoles in his study of the merger of shielded vortices. In a study of tornadoes using contour dynamics, $\operatorname{Lin}^{5}$ observed the formation of triangular vortices via the nonlinear destabilization of shielded monopolar structures. Carnevale and Kloosterziel ${ }^{6}$ have studied the formation, evolution and sta-

\footnotetext{
${ }^{\text {a)} E l e c t r o n i c ~ m a i l: ~ d . c r o w d y @ i c . a c . u k ~}$
}

${ }^{b)}$ Electronic mail: martin.cloke@ic.ac.uk bility, both experimentally and numerically, of various multipolar vortex structures with emphasis on the triangular (quadrupolar) vortex. Their conclusion is that, unlike pentapolar and other higher order structures, the quadrupole proves relatively robust and stable to perturbations of appropriately small amplitude. They also studied a point vortex model of a symmetric triangular vortex and showed that the configuration is stable to finite perturbations in both the equilibrium positions and strengths of the satellites.

Morel and Carton ${ }^{7}$ independently reached similar conclusions on the robustness of the quadrupolar vortex and concluded that they are robust whether initially perturbed or not and can resist certain rather severe deformations. Morel and Carton ${ }^{7}$ remark on the fact that, starting from different initial conditions, their results are in qualitative agreement with those of Carnevale and Kloosterziel. ${ }^{6}$

A class of exact solutions of the two-dimensional Euler equations representing multipolar vortex structures with distributed vorticity has recently been found by Crowdy. ${ }^{1}$ For each $N \geqslant 2$, the analysis of Ref. 1 provides a continuous oneparameter family (parametrized by a real parameter $a$ ) of solutions which, in the limit $a \rightarrow \infty$ become increasingly monopolar in form but which, as $a$ decreases, develop into coherent structures possessing all the qualitative features which are now recognized as characterizing an $(N+1)$-polar vortex. The exact solution class consists of various finite distributions of line vortices superposed on an ambient vortex patch comprising a distributed region of uniform vorticity.

Beyond the realm of multipolar vortices, vortical configurations involving line vortices superposed on a background vorticity patch have recently been the subject of much theoretical and experimental investigation in other contexts. Lin $^{5}$ studies the evolution of a small-scale line vortex superposed on a vortex patch to model a tornado vortex moving in a tornado mesocyclone. In this application, the background patch of vorticity models the tornado mesocy- 
clone while a smaller-scale vortex typically models the tornado cyclone. As discussed by Lin, the "multiple vortex" phenomenon is now well known in the study of tornadoes and consists of a situation in which the tornado core is surrounded by a number of orbiting "suction spots" which are often seen to orbit the core and are the cause of much damage and destruction. It is conceivable that the central line vortex in the solutions of Ref. 1 might model the core of the tornado cyclone (superposed on the ambient vortex patch which models the tornado mesocyclone) with the satellite line vortices modelling the suction spots. Patch/line-vortex systems are also studied because of their relevance in modelling the relaxation of 2D turbulence in which an initially turbulent vorticity distribution coalesces into a number of distinct vorticity maxima effectively superposed on the region of ambient vorticity. ${ }^{8}$ Durkin and Fajans ${ }^{9,10}$ have performed experiments in a Malmberg-Penning trap in an attempt to under the dynamics of "vortex-within-a-vortex" phenomena. Jin and Dubin ${ }^{11}$ later performed an analysis on such a system.

It is of interest to examine the stability properties of the exact multipolar vortices of Ref. 1. This is the subject of the present paper. A convenient feature of the exact solutions of Ref. 1 is that the linear stability properties can be systematically studied as a function of the continuous parameter $a$ for each integer $N \geqslant 2$. This requires a numerical treatment but is considerably simplified by knowledge of closed-form formulas for the base-state solutions.

The contents of the paper are as follows. In Sec. II the relevant formulas for the exact solutions found in Ref. 1 are reviewed. This section also includes a detailed discussion, not included in the original presentation, ${ }^{1}$ of limiting states within this class. Section III presents an analytical treatment of the linear stability of the basic shielded Rankine vortex (which was the starting point for the generalized solutions found in Ref. 1). Then, the linear stability of the general solutions of Ref. 1 is studied using a numerical spectral method based on Taylor and Laurent series. Section IV describes a numerical investigation, using contour dynamics methods, into the nonlinear evolution of the solution class, starting with some preliminary observations on the nonlinear stability of the shielded Rankine vortex. The paper concludes with a discussion of the results (Sec. V).

\section{EXACT SOLUTIONS OF REF. 1}

\section{A. Review}

The solutions in Crowdy ${ }^{1}$ consist of a simply connected patch of uniform vorticity $\omega$ on which a finite distribution of line vortices are superposed such that the global configuration is in dynamic equilibrium. A schematic of a typical quadrupolar vortex is shown in Fig. 1. $\omega$ can be chosen arbitrarily. For any integer $N \geqslant 2$, the shape of the vortex patch is described by a conformal map $z_{0}(\zeta)$ from the unit $\zeta$ disc (in a parametric $\zeta$ plane) to the interior of the patch. The mapping takes the unit circle $|\zeta|=1$ to the boundary of the vortex patch and is given by

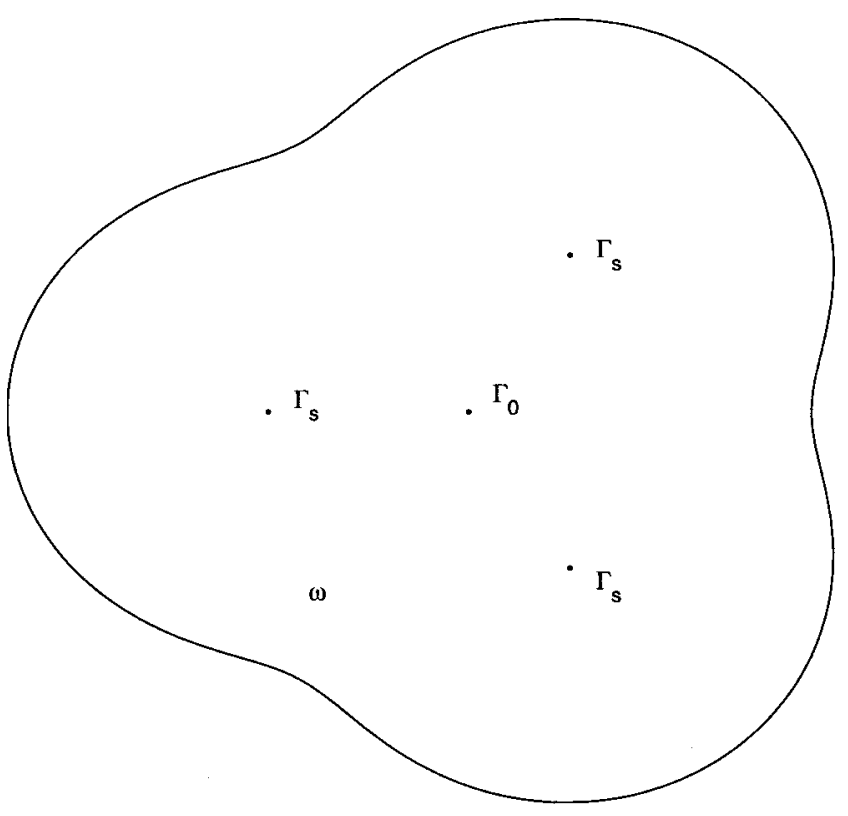

FIG. 1. Schematic of a quadrupolar vortex consisting of a uniform patch of vorticity $\omega$ with four superposed line vortices - a central line vortex of strength $\Gamma_{0}$ and three satellite line vortices each of strength $\Gamma_{s}$.

$$
z_{0}(\zeta)=R \zeta\left(1+\frac{b}{\zeta^{N}-a^{N}}\right),
$$

where $R, a$, and $b$ are suitable real parameters. Given $a$ and $N$, the value of $b$ is the solution of the nonlinear algebraic equation

$$
\begin{aligned}
& \frac{1}{a}\left(1+\frac{b a^{N}}{\left(1-a^{2 N}\right)}\right)-a+\frac{b(N-1)}{2 N a^{N-1}}+\frac{b}{2 N a^{N}} \frac{z_{0 \zeta \zeta}\left(a^{-1}\right)}{z_{0 \zeta}\left(a^{-1}\right)} \\
& \quad=0,
\end{aligned}
$$

an equation that is independent of $R \cdot z_{0 \zeta}(\zeta)$ and $z_{0 \zeta \zeta}(\zeta)$ denote the first and second derivatives of $z_{0}(\zeta)$. This nonlinear equation for $b$ can be rearranged to form a quadratic,

$$
c_{2}(a ; N) b^{2}+c_{1}(a ; N) b+c_{0}(a ; N)=0,
$$

where

$$
\begin{aligned}
c_{2}(a ; N)= & \frac{a}{2 N\left(1-a^{2 N}\right)^{3}}\left[N-1+2 N(1-N) a^{2 N-2}\right. \\
& +\left(2 N^{2}-2 N+2\right) a^{2 N}-2 N a^{4 N-2} \\
& \left.+(N-1) a^{4 N}\right], \\
c_{1}(a ; N)= & \frac{1}{2 a^{N-1} N\left(1-a^{2 N}\right)^{2}}[N-1 \\
& +2 N(2-N) a^{2 N-2}+\left(2 N^{2}-4 N+2\right) a^{2 N} \\
& \left.-4 N a^{4 N-2}+(3 N-1) a^{4 N}\right], \\
c_{0}(a ; N)= & \frac{1-a^{2}}{a},
\end{aligned}
$$

which allows $b(a ; N)$ to be found explicitly as 
$b(a ; N)=\frac{-c_{1}(a ; N)-\sqrt{\left[c_{1}(a ; N)\right]^{2}-4 c_{0}(a ; N) c_{2}(a ; N)}}{2 c_{2}(a ; N)}$.

The second possible solution for $b$ does not yield a conformal map that is a univalent function in the unit $\zeta$ circle and is therefore physically inadmissible. The fact that a closed-form analytical expression for $b(a ; N)$ can be derived is not stated in Crowdy, ${ }^{1}$ but is a significant observation because it renders the exact solution class completely explicit.

$R$ is an arbitrary normalization parameter which determines the size of the vortex structure. Let $\mathcal{A}$ denote the area of the patch. In Ref. $1 R$ is chosen so that $\mathcal{A}=\pi$. Here, a different choice is made; we specify that each of the satellite line vortices is a unit distance from the central line vortex which is at the coordinate origin. Because the map (1) has a satellite line vortex on the negative real axis, we insist that this vortex is in fact at $z=-1$ leading to

$$
R=\frac{a^{2 N+1}-a}{1-a^{2 N}+b a^{N}} .
$$

With this choice, the central line vortex is at $z=0$ while the $N$ satellite line vortices are at

$$
z_{k 0}=-e^{2 \pi i(k-1) / N}, \quad k=1,2, \ldots, N .
$$

The strength $\Gamma_{0}$ of the central line vortex and the strength $\Gamma_{s}$ of each of the satellite line vortices are given by

$$
\begin{aligned}
& \Gamma_{0}=\omega \pi R z_{0 \zeta}(0), \\
& \Gamma_{s}=-\frac{\omega \pi R b z_{0 \zeta}\left(a^{-1}\right)}{N a^{N}} .
\end{aligned}
$$

Let $\Gamma_{p}$ denote the total circulation of the patch of area $\mathcal{A}$ and uniform vorticity $\omega$, then

$$
\Gamma_{p}=\omega \mathcal{A} \text {. }
$$

The fact that the total circulation of the coherent structure is zero implies that the circulations are related via

$$
\Gamma_{p}+\Gamma_{0}+N \Gamma_{s}=0 .
$$

\section{B. Limiting states}

We now discuss the limiting states of the class of exact solutions just described and both clarify and amplify some brief remarks made on this matter in Ref. 1.

The subject of limiting $V$-states has a rich history in the vortex dynamics literature. The term " $V$-state" normally refers to a globally uniform, simply-connected patch of vorticity. ${ }^{12}$ Overman ${ }^{13}$ has performed a study of limiting $V$ states and, by means of a local analysis, has shown that points of nonanalyticity in the boundaries of patches of uniform vorticity can either be $90^{\circ}$ corners or cusps. Overman also examines the literature for equilibrium solutions of the Euler equations involving uniform $V$-states and concludes, using a combination of local and global arguments, that all known solutions exhibit corner formation as the relevant limiting states. At that time, no examples of limiting $V$-state equilibria exhibiting cusps were known. The solutions found in Crowdy, ${ }^{1}$ while consisting of patches of uniform vorticity

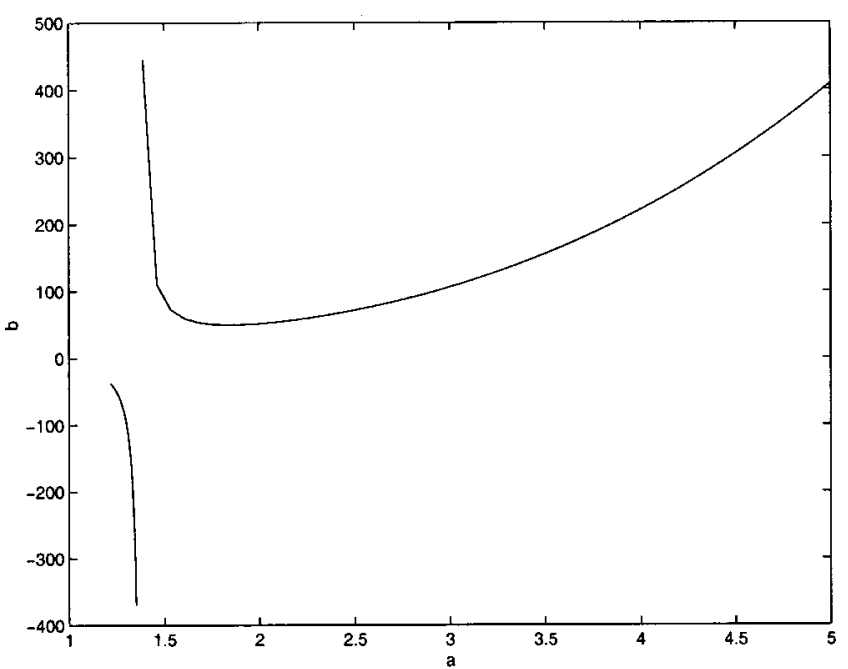

FIG. 2. $b$ against $a$ for $N=3: a_{\text {crit }}^{(3)}=1.211 ; a_{\text {asymp }}^{(3)}=1.371$.

are not, strictly speaking, $V$-states in the sense that the vorticity in the patch is not globally uniform. There is also a finite distribution of superposed line vortices. Nevertheless, the local analysis of Overman which leads to the conclusion that points of nonanalyticity in the boundaries of locally uniform patches of vorticity must be either $90^{\circ}$ corners or cusps still pertains to the solutions of Crowdy ${ }^{1}$ (provided a line vortex does not draw close to the point of nonanalyticity in the limit). It is therefore of interest to examine whether the solutions of Crowdy ${ }^{1}$ are consistent with Overman's analysis.

In a short section of Ref. 1 on limiting vortex shapes, the term "limiting" is used loosely to exhibit the qualitative fact that as the parameter $a$ gets large a monopolar vortex (resembling the shielded Rankine vortex) is obtained while as $a$ draws close to unity multipolar vortex structures having shapes highly reminiscent of some schematical drawings of Carnevale and Kloosterziel ${ }^{6}$ are observed. In Fig. 8 of Ref. 1 some example shapes are depicted for $N=3$ and 4 with $a$ values close to unity but the exact $a$ values used are not given explicitly.

In fact, the $a$ values used in Fig. 8 of Ref. 1 are close to the position of the vertical asymptote in the graphs of $b$ against $a$ which can be clearly seen in Figs. 2 and 3 shown here for the values $N=3$ and 4 . The corresponding patch shapes, similar to those drawn in Fig. 8 of Ref. 1, are reproduced here in Fig. 4. In Ref. 1 the graph of $b$ against $a$ was depicted only for $N=2$ and it is stated there that the graphs for $N \geqslant 3$ are qualitatively similar. A comparison of Figs. 2 and 3 of the present paper with Fig. 2 of Ref. 1 reveals that this is indeed true provided one only looks to the right of a vertical asymptote which appears at a value of $a$ which is strictly greater than 1 and which will henceforth be denoted $a_{\text {asymp }}^{(N)}$. Note that for $N=2$, the case considered in most detail in Ref. 1 this vertical asymptote occurs at $a=1$ while for all $N \geqslant 3$ this asymptote occurs at some $a_{\text {asymp }}^{(N)}>1$. Thus for $N \geqslant 3$ there is an additional branch of possible $b$ values (shown explicitly in Figs. 2 and 3) which are negative but which also give rise to univalent conformal mappings from 


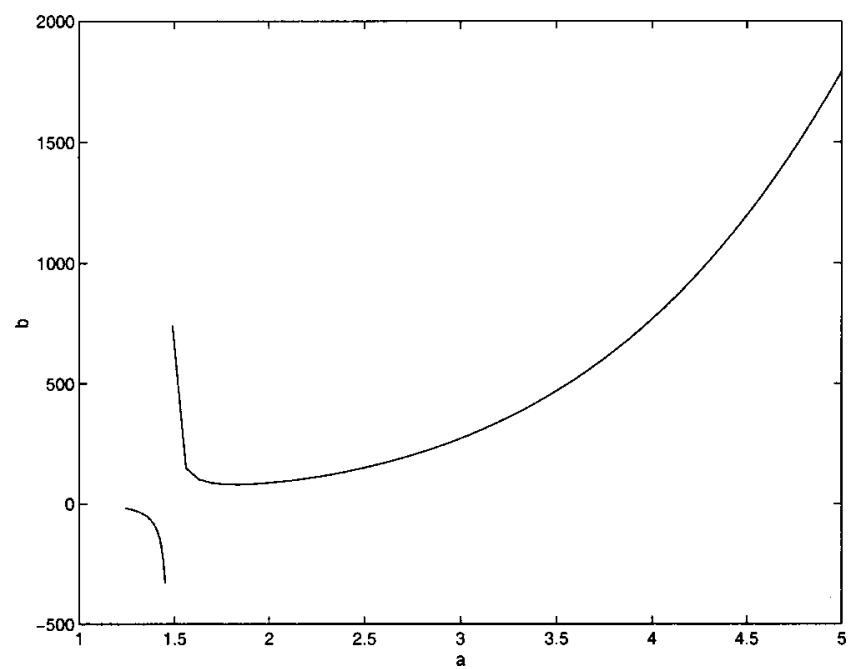

FIG. 3. $b$ against $a$ for $N=4: a_{\text {crit }}^{(4)}=1.241 ; a_{\text {asymp }}^{(4)}=1.477$.

the unit $\zeta$ disc (this additional branch, which exists for $N$ $\geqslant 3$ but not $N=2$, was missed at the time of writing Ref. 1 ).

The vertical asymptote in the $b$-against- $a$ graphs for $N$ $\geqslant 3$ does not represent a genuine singularity in the class of exact solutions of Ref. 1 and the corresponding solutions are not limiting states. A hint that this is the case is given by the graphs of $\Gamma_{0} / \Gamma_{p}$ and $\Gamma_{s} / \Gamma_{p}$ as $a$ passes through $a_{\text {asymp }}^{(N)}$. These graphs are shown in Figs. 5 and 6 for $N=3$ and 4. The key to understanding this artificial singularity is to note that the value of $\Gamma_{0}$ at $a_{\text {asymp }}^{(N)}$ is equal to zero. Thus the solution at $a=a_{\text {asymp }}^{(N)}$ for $N \geqslant 3$ corresponds to seeking solutions consisting of a finite-area vortex patch with $N$ superposed satellite line vortices but no central line vortex. It is easy to extend the derivation of Ref. 1 to show that the corresponding conformal map from the unit $\zeta$ circle in this case would have the modified form

$$
\widetilde{z}_{0}(\zeta)=\frac{R \zeta}{\zeta^{N}-a^{N}}
$$

To find $a_{\text {asymp }}^{(N)}$ it is simply necessary to solve the modified nonlinear equation

$$
\frac{a^{N-1}}{1-a^{2 N}}+\frac{(N-1)}{2 N a^{N-1}}+\frac{1}{2 N a^{N}} \frac{\widetilde{z}_{0 \zeta \zeta}\left(a^{-1}\right)}{\widetilde{z}_{0 \zeta}\left(a^{-1}\right)}=0 .
$$

This equation depends only on the parameter $a$ and it is found to have a unique solution in the range $[1, \infty)$. Note that
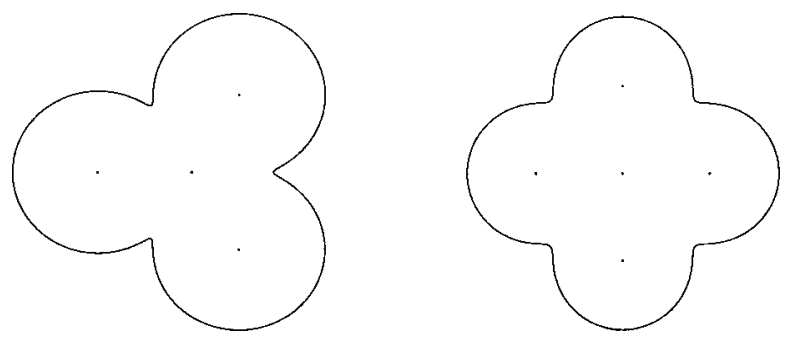

FIG. 4. Vortex patch shapes for $a$ close to $a_{\text {asymp }}^{(N)}: N=3, a=1.385 ; N=4$, $a=1.485$ (cf. Fig. 8 of Ref. 1). Line vortices are shown as bold dots.

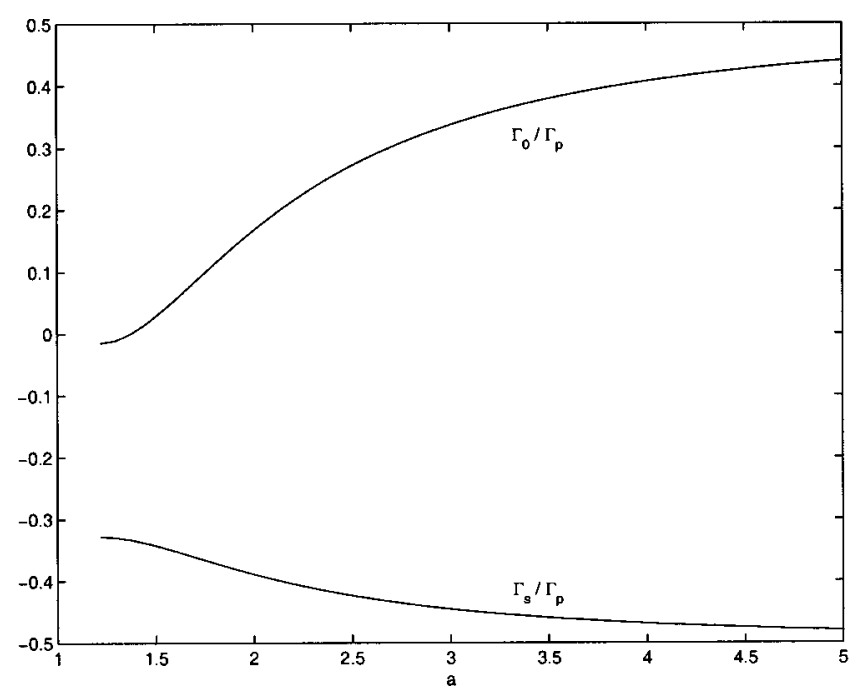

FIG. 5. Graph of $\Gamma_{0} / \Gamma_{p}$ and $\Gamma_{s} / \Gamma_{p}$ against $a$ for $N=3$.

(12) can be derived as a formal limit of (2) as $b \rightarrow \infty$. For each $N$ the solution of (12) will be denoted $a_{\text {asymp }}^{(N)}$. The first few values are calculated to be

$$
a_{\text {asymp }}^{(3)}=1.371, \quad a_{\text {asymp }}^{(4)}=1.477, \quad a_{\text {asymp }}^{(5)}=1.507 .
$$

It is clear from Figs. 2 and 3 that the positions of the vertical asymptotes of the $b$-against $a$ graphs for $N=3$ and $N=4$ coincide with $a_{\text {asymp }}^{(3)}$ and $a_{\text {asymp }}^{(4)}$, respectively.

As a remark, Crowdy ${ }^{15}$ has shown that there exists a branch of multipolar equilibria consisting of doubly connected regions of vorticity which bifurcate from the steady solutions (11) with $a=a_{\text {asymp }}^{(N)}$ for each $N \geqslant 3$. These solutions are parametrized by a parameter $\rho$ which, when equal to zero, give the solutions (11) with $a=a_{\text {asymp }}^{(N)}$. For $\rho>0$, a small region of quiescent irrotational fluid centered at the origin begins to develop thus forming an annulus of vorticity containing $N$ line vortex singularities.

Having better understood the vertical asymptotes in the $b$-against- $a$ graphs for $N \geqslant 3$, the question arises as to exactly

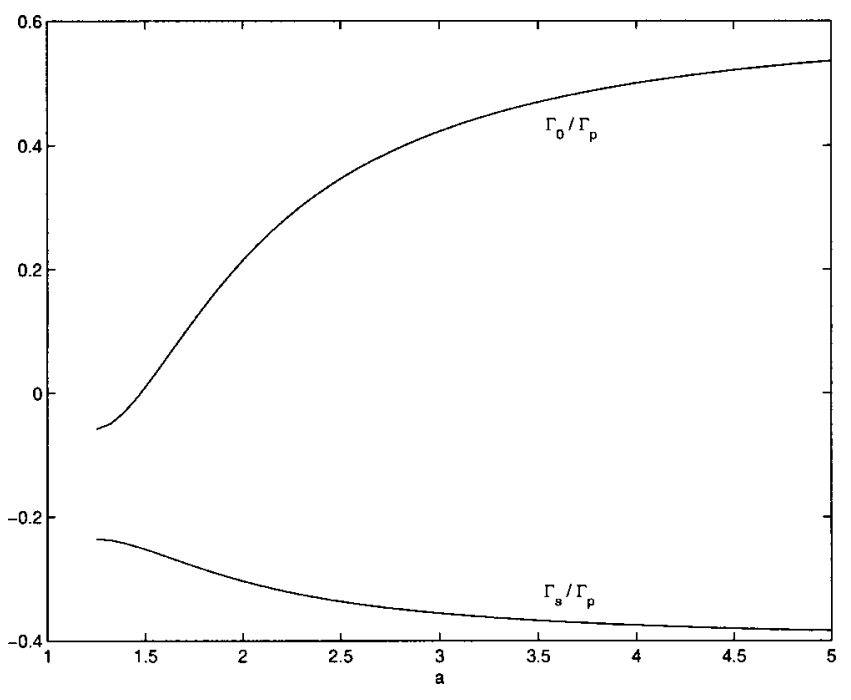

FIG. 6. Graph of $\Gamma_{0} / \Gamma_{p}$ and $\Gamma_{s} / \Gamma_{p}$ against $a$ for $N=4$. 
how close to 1 the parameter $a$ can be made while still giving an exact equilibrium solution of Euler. Indeed, reducing $a$ below $a_{\text {asymp }}^{(N)}$ should now provide the limiting states in the same sense used by Overman. ${ }^{13}$

It turns out that, for each $N \geqslant 3$, a critical value of the parameter $a$ exists at which $N$ cusps form simultaneously at $N$ distinct points on the boundary of the vortex patch. The critical $a$ value in each case will be denoted $a_{\text {crit }}^{(N)}$. For a given $N$, exact solutions exist for any value of the continuous real parameter $a$ in the range

$$
a \in\left[a_{\text {crit }}^{(N)}, \infty\right) .
$$

To find $a_{\text {crit }}^{(N)}$ it is necessary to solve the stationarity condition (2) and the equation

$$
z_{0 \zeta}\left(e^{\pi i / N}\right)=0
$$

simultaneously for $a_{\text {crit }}^{(N)}$ and the corresponding $b_{\text {crit }}^{(N)}$. These two nonlinear equations can be solved numerically using Newton's method (or, indeed, solved analytically). The solutions for the first few values of $N$ are found to be

$$
a_{\text {crit }}^{(3)}=1.211, \quad a_{\text {crit }}^{(4)}=1.241, \quad a_{\text {crit }}^{(5)}=1.232 .
$$

Recall that a necessary condition for (1) to represent a univalent conformal map from the unit $\zeta$ disc to the vortex patch is that $z_{0 \zeta}$ vanishes nowhere inside $|\zeta| \leqslant 1$. A zero impinging on the unit circle will lead to a loss of univalency of the conformal map and will therefore constitute a limiting state. By symmetry, the limiting case of the conformal map (1) corresponds to $N$ simple zero of $z_{0 \zeta}$ simultaneously impinging on the unit $\zeta$ circle. This gives rise to the simultaneous formation of $N$ cusps in the boundary of the vortex patch. The critical patch shapes for $N=3,4$, and 5 are shown in Fig. 7.

For situation for $N=2$ is different and is the case considered in most detail in Ref. 1. It is found that $a$ can draw arbitrarily close to 1 and, in this limit, the corresponding patch resembles two touching circular discs while the two satellite line vortices tend towards the center of each of these two discs. The solution for $a=1.05$ is shown in Fig. 8. There is a line vortex at the center of the configuration at the point to which the two cusps in the patch boundary are tending. Strictly speaking, care is needed in applying the local analysis of Overman to this case because, in the limit, the point of nonanalyticity of the vortex boundary coincides with a line vortex singularity. However, the strength of this line vortex tends to zero in the limit and two cusps are still seen to form in the boundary of the patch. We do not investigate this point in any detail. A class of rotating $V$-states exhibiting cusp singularities in their limiting configurations has recently been found by Crowdy. ${ }^{14}$ A detailed local analysis of both the limiting patch shape and velocity field is performed in Ref. 14 and, while we omit the details, a similar analysis can be performed for the exact solution class of interest here.

If the central line vortex is removed, the vortex configuration in Fig. 8 resembles two touching shielded Rankine vortices. The general idea of combining a disjoint collection of shielded Rankine vortices (which do not interact if they do not overlap) to form more complicated global equilibria of
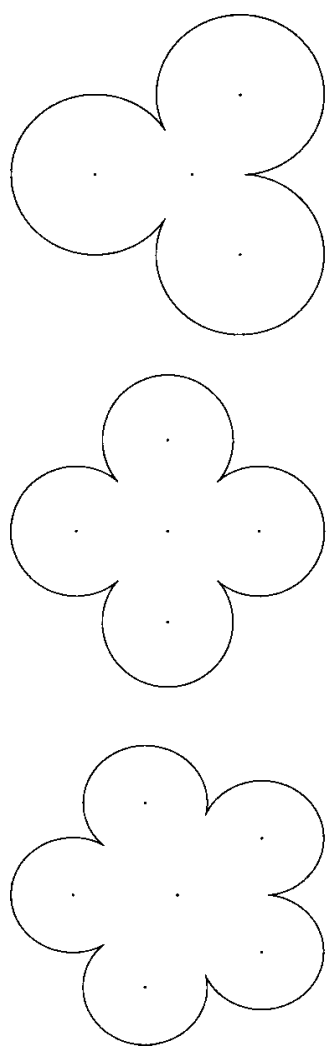

FIG. 7. Vortex patch shapes for $a$ close to $a_{\text {crit }}^{(N)}: N=3, a_{\text {crit }}^{(3)}=1.211 ; N$ $=4, a_{\text {crit }}^{(4)}=1.241 ; N=5, a_{\text {crit }}^{(5)}=1.232$. These are close to the limiting states in the sense of Overman (Ref. 13) and exhibit near-cusp singularities in the patch boundary. Line vortices are shown as bold dots.

the two-dimensional Euler equation proves to be a fruitful one. Crowdy ${ }^{15,16}$ has shown that there are situations in which a number of initially disjoint shielded Rankine vortices can draw into contact and be "continued" to form higher-order compound vortices. The reader is referred to Refs. 15 and 16 for a development of these ideas.

\section{LINEAR STABILITY}

\section{A. Shielded Rankine vortex}

Flierl $^{2}$ has examined the linear stability of a zero total circulation monopolar structure consisting of a unit circular disc $r \leqslant 1$ of unit positive vorticity shielded by an annular patch of uniform vorticity $-q$ for radii between $1<r<d$. It is found that as the width of the outer annulus decreases, the wave number of the most linearly unstable mode also increases.

The shielded Rankine vortex can be understood as a limit of Flierl's configuration; the outer radius $d$ of the annu-

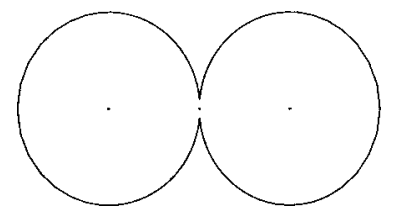

FIG. 8. Patch boundary for $N=2$ and $a=1.05$. Line vortices are shown as bold dots. 
lus is rescaled to unity while the area of the enclosed circular patch is reduced to zero while simultaneously increasing its vorticity to infinity in such a way as to keep the total circulation of the enclosed vortex constant. In spite of this, it is not particularly straightforward to compute the corresponding limit of Flierl's linear stability results. Therefore, we now proceed to use rather separate analytical methods to explicitly study the linear stability of the shielded Rankine vortex solution. This analysis is useful for two reasons: first, it will provide a check on the numerical code written to find the linear stability spectrum in the general case; second, it is instructive in explaining the general numerical method to be employed later.

The steady shielded Rankine vortex solution can be written

$$
u-i v= \begin{cases}-\frac{i \omega}{2}\left(\bar{z}-\frac{1}{z}\right), & z \in D, \\ 0, & z \notin D .\end{cases}
$$

Under a general (irrotational) perturbation, the Helmholtz vortex theorems ${ }^{18}$ can be used to deduce that the velocity field associated with the perturbed vortex can be written as

$$
u-i v= \begin{cases}-\frac{i \omega}{2}\left(\bar{z}-\frac{1}{z-\hat{z}_{0}(t)}+F(z, t)\right), & z \in D(t), \\ -\frac{i \omega}{2} G(z, t), & z \notin D(t),\end{cases}
$$

where $D(t)$ now denotes the perturbed patch and $F(z, t)$ and $G(z, t)$ are functions analytic inside and outside $D(t)$, respectively. $\hat{z}_{0}(t)$ denotes the (perturbed) position of the central line vortex. The dynamic pressure condition is equivalent to continuity of the velocity field on the patch boundary. This implies

$$
F(z, t)-G(z, t)=-\bar{z}+\frac{1}{z-\hat{z}_{0}(t)} \quad \text { on } \partial D(t) .
$$

Equation (19) constitutes a Riemann-Hilbert problem for the analytic functions $F(z, t)$ and $G(z, t)$.

We now assume that the perturbations are small so that their evolution can be described within linear theory. The base-state conformal map in this case is simply

$$
z_{0}(\zeta)=\zeta
$$

It is now conjectured that the linear eigenmodes have the functional form $a_{n}(t) \zeta^{n}$ for $n \geqslant 1$ where $a_{n}(t) \in \mathbb{C}$ is an order one quantity so that the perturbed conformal map has the form

$$
z(\zeta, t)=z_{0}(\zeta)+\epsilon a_{n}(t) \zeta^{n}+\mathcal{O}\left(\epsilon^{2}\right), \quad n \geqslant 1 .
$$

The perturbed position of the central line vortex is assumed to have the form

$$
\hat{z}_{0}(t)=\epsilon b(t),
$$

where $b(t) \in \mathbb{C}$ is an order one quantity.

We now verify that this ansatz for the linear eigenmodes is correct. First consider (19). Using (21) and (22) in (19) it is seen

$$
\begin{aligned}
F(z, t)-G(z, t)= & -\frac{1}{\zeta}-\frac{\epsilon \bar{a}_{n}}{\zeta^{n}}+\frac{1}{\zeta+\epsilon a_{n}(t) \zeta^{n}-\epsilon b} \\
& +\mathcal{O}\left(\epsilon^{2}\right) .
\end{aligned}
$$

Expanding correction to $\mathcal{O}(\epsilon)$,

$$
F(z, t)-G(z, t)=\epsilon\left(-\frac{\bar{a}_{n}}{\zeta^{n}}+\frac{b}{\zeta^{2}}-a_{n} \zeta^{n-2}\right)+\mathcal{O}\left(\epsilon^{2}\right) .
$$

The first two terms on the right-hand side are clearly not analytic inside the patch. Indeed, it is deduced that

$$
F(z(\zeta, t))=-\epsilon a_{n}(t) \zeta^{n-2},
$$

which is analytic inside the patch.

The kinematic boundary condition can be written in the form

$$
\operatorname{Re}\left[\frac{z_{t}(\zeta, t) \bar{z}_{\zeta}\left(\zeta^{-1}, t\right)}{\zeta}\right]=\operatorname{Re}\left[\frac{(u+i v) \bar{z}_{\zeta}\left(\zeta^{-1}, t\right)}{\zeta}\right] .
$$

To proceed, it is assumed that all perturbed quantities have a time dependence of the form $e^{\sigma t}$ where $\sigma$ is an eigenvalue which remains to be determined. In particular,

$$
\begin{aligned}
& a_{n}(t)=\widetilde{a}_{n} e^{\sigma t}, \quad b(t)=\widetilde{b} e^{\sigma t}, \quad \bar{a}_{n}(t)=\widetilde{a}_{n}^{*} e^{\sigma t}, \\
& \bar{b}(t)=\widetilde{b}^{*} e^{\sigma t},
\end{aligned}
$$

where $\widetilde{a}_{n}, \tilde{b}, \widetilde{a}_{n}^{*}, \tilde{b}^{*}$ are complex constants. The coefficients $\tilde{a}_{n}^{*}$ and $\tilde{b}^{*}$ are $\tilde{\sigma}_{\text {not }}$ assumed to be the actual complex conjugates of $\tilde{a}_{n}$ and $\tilde{b}$. To clarify notation, the actual complex conjugate of $\tilde{a}_{n}$ will be denoted $\overline{\tilde{a}}_{n}$. Henceforth, $\tilde{a}_{n}$ and $\tilde{a}_{n}^{*}$ are independent complex parameters, as are $\tilde{b}$ and $\tilde{b}^{*}$. This technique is also used in Meiron, Saffman and Schatzman ${ }^{17}$ in their study of the linear stability of inviscid vortex streets. A physically realistic perturbation can be constructed from the solutions obtained in this way.

Using (21), (22), and (25) in (26) to leading order we obtain (dropping tildes)

$$
\begin{aligned}
\sigma a_{n} \zeta^{n-1}+\frac{\sigma a_{n}^{*}}{\zeta^{n-1}}= & -\frac{i \omega}{2}\left(\frac{a_{n}^{*}}{\zeta^{n-1}}-\frac{b}{\zeta}\right) \\
& +\frac{i \omega}{2}\left(a_{n} \zeta^{n-1}-b^{*} \zeta\right) .
\end{aligned}
$$

First consider $n=1$. In this case, equating coefficients of $\zeta^{0}$ and $\zeta^{ \pm 1}$ in (28) we deduce

$$
\sigma\left(a_{1}+a_{1}^{*}\right)=-\frac{i \omega}{2}\left(a_{1}^{*}-a_{1}\right), \quad b=0, \quad b^{*}=0 .
$$

This gives two (dynamically unimportant) modes: one corresponding to a neighboring steady state in which the radius of the vortex patch has increased slightly $(\sigma=0)$ and another to a rotational degree of freedom which is removed by insisting $a_{1}^{*}=a_{1}$.

Now consider $n=2$. In this case, comparing coefficients of $\zeta^{ \pm 1}$ we obtain the two equations 


$$
\begin{gathered}
\sigma a_{2}=\frac{i \omega}{2}\left(a_{2}-b^{*}\right), \\
\sigma a_{2}^{*}=-\frac{i \omega}{2}\left(a_{2}^{*}-b\right) .
\end{gathered}
$$

Note that because $a_{2}, a_{2}^{*}, b$, and $b^{*}$ are assumed to be independent, Eqs. (30) constitute two equations for four independent variables. We therefore require two more equations. These follow from the dynamical equations for the evolution of the central line vortex which we have yet to write down. By the Helmholtz vortex theorems, ${ }^{18}$ it is known that a line vortex moves with the non-self-induced component of the local velocity field. In this case, this can be shown to be equivalent to the (linearized) equation

$$
\sigma b^{*}=-\frac{i \omega}{2}\left(b^{*}-a_{2}\right) .
$$

This equation, together with its complex conjugate, provide the two additional equations needed to supplement (30). These equations lead to a standard eigenvalue problem for a $4 \times 4$ matrix all of whose eigenvalues are found to be zero.
For all $n \geqslant 3$, equating coefficients of $\zeta^{ \pm(n-1)}$ and $\zeta^{ \pm 1}$ in (26) yields

$$
\sigma a_{n}=\frac{i \omega a_{n}}{2}, \quad \sigma a_{n}^{*}=-\frac{i \omega a_{n}^{*}}{2}, \quad b=0, \quad b^{*}=0,
$$

which yields two eigenvalues $\pm i \omega / 2$. Each of these two eigenvalues has infinite multiplicity. The linear stability spectrum of the shielded Rankine vortex therefore consists of five zero eigenvalues and an infinitude of $\pm i \omega / 2$ pairs. Only the $n=2$ shape perturbation mode interacts with the line vortex evolution. It is concluded that the shielded Rankine vortex is neutrally stable to small perturbations (i.e., there are no linear eigenmodes with eigenfrequencies having strictly positive real part). The nonlinear stability of the shielded Rankine vortex is examined briefly in Sec. III.

\section{B. General equilibria of Ref. 1}

Using Helmholtz laws ${ }^{18}$ the velocity field associated with general irrotational perturbations to the general class of equilibria found in Ref. 1 can be written

$$
u-i v=\left\{\begin{array}{l}
-\frac{i \omega}{2}\left(\bar{z}-\sum_{k=1}^{N} \frac{\gamma_{s}}{z-z_{k}(t)}-\frac{\gamma_{0}}{z-z_{0}(t)}-F(z, t)\right), \quad z \in D(t), \\
-\frac{i \omega}{2} G(z, t), \quad z \notin D(t),
\end{array}\right.
$$

where

$$
\begin{gathered}
\gamma_{0}=-R z_{0 \zeta}(0), \\
\gamma_{s}=\frac{R b z_{0 \zeta}\left(a^{-1}\right)}{N a^{N}},
\end{gathered}
$$

and where $D(t)$ is the perturbed vortex patch, $F(z, t)$ is analytic inside $D(t)$ and $G(z, t)$ is analytic outside $D(t)$ and is $\mathcal{O}(1 / z)$ as $|z| \rightarrow \infty$. The set of complex functions of time $\left\{z_{k}(t) \mid k=0, \ldots, N\right\}$ denotes the perturbed positions of the central and satellite line vortices. Continuity of velocity on the vortex jump requires that

$$
\begin{aligned}
F(z, t)-G(z, t)= & -\bar{z}+\sum_{k=1}^{N} \frac{\gamma_{s}}{z-z_{k}(t)} \\
& +\frac{\gamma_{0}}{z-z_{0}(t)} \text { on } \partial D(t) .
\end{aligned}
$$

Equation (35) is a standard Riemann-Hilbert problem. The solution for $F(z, t)$ is given by

$$
\begin{aligned}
F(z, t)= & \frac{1}{2 \pi i} \oint_{\partial D(t)}\left(-\bar{z}^{\prime}+\sum_{k=1}^{N} \frac{\gamma_{s}}{z^{\prime}-z_{k}(t)}\right. \\
& \left.+\frac{\gamma_{0}}{z^{\prime}-z_{0}(t)}\right) \frac{d z^{\prime}}{z^{\prime}-z} \\
= & -\frac{1}{2 \pi i} \oint_{\partial D(t)} \frac{\bar{z}^{\prime} d z^{\prime}}{z^{\prime}-z}, \quad z \in D(t),
\end{aligned}
$$

the second equality following because all the points $\left\{z_{k}(t) \mid k=0,1, \ldots, N\right\}$ are inside $D(t)$.

The linearly perturbed conformal map, and its conjugate function, are assumed to be of the form

$$
\begin{aligned}
& z(\zeta, t)=z_{0}(\zeta)+\epsilon e^{\sigma t} \hat{z}(\zeta), \\
& \overline{z(\zeta, t)}=\overline{z_{0}(\zeta)}+\epsilon e^{\sigma t} \bar{z}(\zeta),
\end{aligned}
$$

where $\epsilon \ll 1$ is a small parameter. Perturbations to the line vortex positions are denoted

$$
z_{k}(t)=z_{k 0}+\epsilon e^{\sigma t} \hat{z}_{k}, \quad k=0, \ldots, N .
$$

The circulations of the line vortices do not change under perturbation. The functions $\hat{z}(\zeta)$ have expansions of the form

$$
\hat{z}(\zeta)=\sum_{n=0}^{\infty} \hat{a}_{n} \zeta^{n}, \quad \overline{\hat{z}}\left(\zeta^{-1}\right)=\sum_{n=0}^{\infty} \frac{\hat{a}_{n}^{*}}{\zeta^{n}},
$$


where $\left\{\hat{a}_{n}^{*}\right\}$ are assumed independent of $\left\{\hat{a}_{n}\right\}$. The expansion (39) is truncated at order $(\mathcal{N} / 2)-1$ where $\mathcal{N}$ is an even integer. The choice of $\mathcal{N}$ is discussed shortly.

Defining the quantities $\zeta_{k 0}$ to be the preimages in the $\zeta$ plane of the points $\left\{z_{k 0}\right\}$, i.e.,

$$
z_{k 0}=z_{0}\left(\zeta_{k 0}\right), \quad k=0, \ldots, N,
$$

then the quantities $\left\{\hat{\zeta}_{k} \mid k=0 \cdots N\right\}$ are defined via the equations

$$
z_{k}(t)=z_{k 0}+\epsilon e^{\sigma t} \hat{z}_{k} \equiv z\left(\zeta_{k 0}+\epsilon \hat{\zeta}_{k}, t\right), \quad k=0, \ldots, N .
$$

To leading order in $\epsilon$,

$$
\hat{z}_{k}=\hat{\zeta}_{k} z_{0 \zeta}\left(\zeta_{k 0}\right)+\hat{z}\left(\zeta_{k 0}\right), \quad k=0, \ldots, N .
$$

Similarly,

$$
\overline{\hat{z}_{k}}=\hat{\zeta}_{k}^{*} z_{0 \zeta}\left(\bar{\zeta}_{k 0}\right)+\overline{\hat{z}\left(\zeta_{k 0}\right)}, \quad k=0, \ldots, N .
$$

Here the set $\left\{\hat{\zeta}_{k}^{*} \mid k=0, \ldots, N\right\}$ is again assumed independent of the set $\left\{\hat{\zeta}_{k} \mid k=0, \ldots, N\right\}$.

The Riemann mapping theorem provides the freedom to specify that

$$
z(0, t)=0
$$

so that $a_{0}=a_{0}^{*}=0$, while to eliminate a rotational degree of freedom we impose $a_{1}=a_{1}^{*}$. After truncation, the total set of unknowns is then

$$
\begin{aligned}
& \left\{\hat{a}_{k} \mid k=1 \cdots \frac{\mathcal{N}}{2}-1\right\}, \quad\left\{\hat{a}_{k}^{*} \mid k=2 \cdots \frac{\mathcal{N}}{2}-1\right\}, \\
& \left\{\hat{\zeta}_{k} \mid k=0 \cdots N\right\}, \quad\left\{\hat{\zeta}_{k}^{*} \mid k=0 \cdots N\right\} .
\end{aligned}
$$

A set of $\mathcal{N}+2 N-1$ equations is required to determine these unknowns.

By composition of analytic functions, we can define $\mathcal{F}_{0}(\zeta)$ via

$$
\mathcal{F}_{0}(\zeta) \equiv F_{0}\left(z_{0}(\zeta)\right)
$$

and similarly $\hat{\mathcal{F}}(\zeta)$ via

$$
F(z(\zeta, t), t)=\mathcal{F}_{0}(\zeta)+\epsilon \hat{\mathcal{F}}(\zeta) e^{\sigma t}+\mathcal{O}\left(\epsilon^{2}\right)
$$

To find $\hat{\mathcal{F}}(\zeta)$, first note that because $F(z, t)$ is analytic inside $D(t)$ then it has a Taylor series of the form

$$
F(z, t)=\sum_{k=0}^{\infty} F_{k} z^{k}
$$

It can be shown from (36) that

$$
F_{k} \equiv-\frac{1}{2 \pi i} \oint_{\partial D} \frac{\bar{z}^{\prime}}{z^{\prime k+1}} d z^{\prime}, \quad k=0,1,2, \ldots .
$$

These coefficients depend on $\epsilon$. Linearizing for small $\epsilon$, so that

$$
F_{k}=F_{k 0}+\epsilon \hat{F}_{k} e^{\sigma t}+\mathcal{O}\left(\epsilon^{2}\right),
$$

it follows that

$$
F_{k 0}=-\frac{1}{2 \pi i} \oint_{\partial D} \frac{\bar{z}_{0}\left(\zeta^{-1}\right) z_{0 \zeta}(\zeta)}{z_{0}(\zeta)^{k+1}} d \zeta
$$

and

$$
\begin{aligned}
\hat{F}_{k}= & -\frac{1}{2 \pi i}\left[\oint_{|\zeta|=1} \frac{\overline{\hat{z}}\left(\zeta^{-1}\right) z_{0 \zeta}(\zeta)}{z_{0}(\zeta)^{k+1}} d \zeta\right. \\
& -\oint_{|\zeta|=1}(k+1) \frac{\bar{z}_{0}\left(\zeta^{-1}\right) z_{0 \zeta}(\zeta) \hat{z}(\zeta)}{\left(z_{0}(\zeta)\right)^{k+2}} d \zeta \\
& \left.+\oint_{|\zeta|=1} \frac{\bar{z}_{0}\left(\zeta^{-1}\right) \hat{z}_{\zeta}(\zeta)}{z_{0}(\zeta)^{k+1}} d \zeta\right] .
\end{aligned}
$$

Some algebra reveals that, to leading order in $\epsilon$, the linearized kinematic boundary condition reduces to the simplified form

$$
\begin{aligned}
\sigma \operatorname{Re}\left[\frac{\bar{z}_{0 \zeta}\left(\zeta^{-1}\right) \hat{z}(\zeta)}{\zeta}\right] \\
=\operatorname{Re}\left[\frac { i \omega } { 2 } \zeta z _ { 0 } \zeta \left(\sum_{k=1}^{N} \frac{\gamma_{s} \hat{z}_{k}}{\left(z_{0}(\zeta)-z_{k 0}\right)^{2}}\right.\right. \\
\left.\left.+\frac{\gamma_{0} \hat{z}_{0}}{\left[z_{0}(\zeta)\right]^{2}}-\sum_{k=0}^{\infty} \hat{F}_{k} z_{0}(\zeta)^{k}\right)\right] .
\end{aligned}
$$

Equation (53) is linear in the perturbation quantities with coefficients which depend nonlocally on the base-state equilibrium. To find equations for the unknowns, the representations (39), (42), and (43) are substituted into (53) and the equation expanded as a Laurent series in $\zeta$ between $\zeta^{-(\mathcal{N} / 2)+2}$ and $\zeta^{(\mathcal{N} / 2)-2}$. The infinite sum appearing in (53) is truncated, consistently, after the $k=(\mathcal{N} / 2)-2$ term. Equating coefficients on both sides of (53) then provides $\mathcal{N}-3$ equations.

The fact that the line vortices are convected with the (non-self-induced) local velocity provides the additional equations

$$
\begin{aligned}
\sigma \overline{\hat{z}_{k}}= & -\frac{i \omega}{2}\left(\overline{\hat{z}_{k}}-\sum_{j \neq k} \frac{\gamma_{s}\left(\hat{z}_{j}-\hat{z}_{k}\right)}{\left(z_{k 0}-z_{j 0}\right)^{2}}\right. \\
& \left.-\frac{\gamma_{0}\left(\hat{z}_{0}-\hat{z}_{k}\right)}{\left(z_{k 0}\right)^{2}}+\hat{F}_{k}^{(l v)}\right), \quad k=1, \ldots, N, \\
\sigma \overline{z_{0}} & =-\frac{i \omega}{2}\left(\overline{z_{0}}-\sum_{k=1}^{N} \frac{\gamma_{s}\left(\hat{z}_{k}-\hat{z}_{c}\right)}{\left(z_{k 0}\right)^{2}}+\hat{F}_{0}^{(l v)}\right),
\end{aligned}
$$

where

$$
\begin{aligned}
\hat{F}_{k}^{(l v)=} & -\frac{1}{2 \pi i} \oint_{|\zeta|=1} \frac{\overline{\hat{z}}\left(\zeta^{-1}\right) z_{0 \zeta}(\zeta)}{z_{0}(\zeta)-z_{k 0}} d \zeta \\
& -\frac{1}{2 \pi i} \oint_{|\zeta|=1} \frac{\bar{z}_{0}\left(\zeta^{-1}\right) \hat{z}_{\zeta}(\zeta)}{z_{0}(\zeta)-z_{k 0}} d \zeta \\
& -\frac{1}{2 \pi i} \oint_{|\zeta|=1} \frac{\bar{z}_{0}\left(\zeta^{-1}\right) z_{0 \zeta}(\zeta)\left(\hat{z}_{k}-\hat{z}(\zeta)\right)}{\left(z_{0}(\zeta)-z_{k 0}\right)^{2}} d \zeta,
\end{aligned}
$$

for $k=0,1, \ldots, N$. The $N+1$ equations (54), together with their complex conjugates, provide the additional $2 N+2$ equations required for a closed, consistent linear system. 


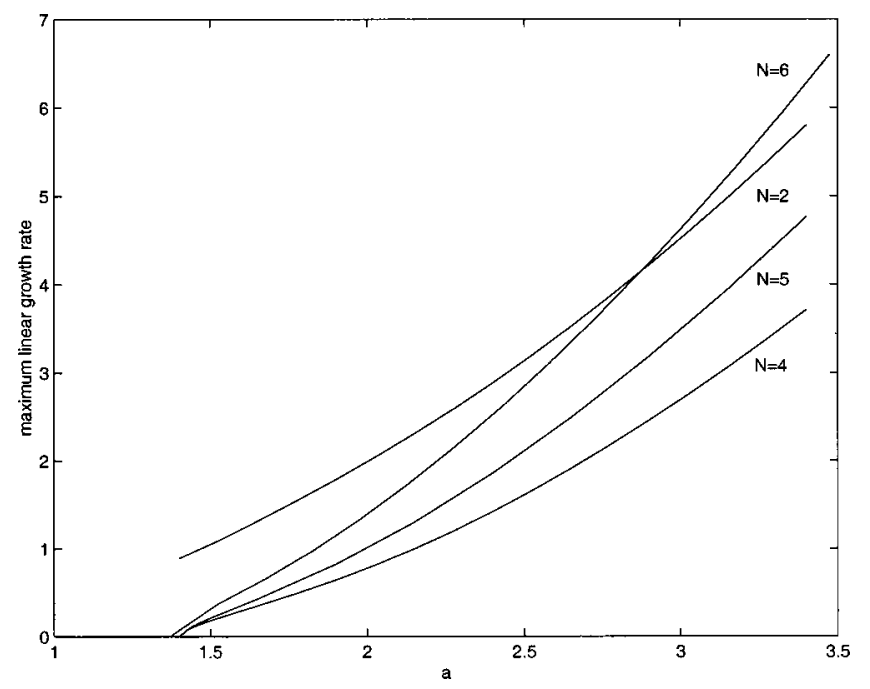

FIG. 9. Maximum linear growth rate against $a$ for $N=2,4,5$, and 6 . The configurations for $N=4,5$, and 6 are found to become neutrally stable at $a_{\text {stab }}^{(4)}=1.40, a_{\text {stab }}^{(5)}=1.41$, and $a_{\text {stab }}^{(6)}=1.37$.

The set of unknowns (45) is vectorized to produce a vector $\mathbf{x}$. The $\mathcal{N}-1+2 N$ equations derived above lead to a generalized eigenvalue problem of the form

$$
\mathbf{A x}=\sigma \mathbf{B x},
$$

where the matrices $\mathbf{A}$ and $\mathbf{B}$ depend purely on the (exact) base-state equilibrium. To find these matrices, all Taylor/ Laurent coefficients of functions of the base-state conformal map (and its derivatives and integrals) are computed by evaluating these functions at $\mathcal{M}$ points on the unit $\zeta$ circle and using fast-Fourier transforms. The following choices are made; $\mathcal{N}$ is taken to be a power of 2 and $\mathcal{M}$ is taken to be at least two powers of 2 higher (typically $\mathcal{M}=4 \mathcal{N}$ ) in order to avoid unacceptable aliasing errors. The accuracy of all calculations is checked by independently altering $\mathcal{N}$ and $\mathcal{M}$. Most of the results which follow use $\mathcal{N}=64$ and $\mathcal{M}=256$.

\section{Checks on the linear stability analysis}

It is known that the dynamics of point vortices and patches of uniform vorticity is Hamiltonian. ${ }^{18}$ Therefore, any eigenvalues that do not occur as pure-real or pure-imaginary pairs will occur in complex conjugate quartets. This provides a first check on the numerical code.

For any steady state, there exists a neighboring equilibrium corresponding to a different value of $R$, i.e., an equilibrium with a slightly different patch area. In addition, we expect two additional zero eigenvalues associated with perturbations which shift the vortex centroid of the vortical structure in either of the two coordinate directions. For any $a$ and $N$, we therefore expect to find at least three zero eigenvalues. This is found to be the case. These modes are recognized and discarded-only perturbations which preserve the area and centroid of the vortex patch are considered.

As an important check on the general eigenmodes corresponding to nonzero eigenvalues, the linear perturbation of the patch area and patch centroid for these modes are computed and it is verified that these perturbations are indeed zero (to within the accuracy of the numerical method). It is also known that angular momentum is another quantity which is conserved by the linearized Euler equations and an additional check is provided by verifying that all modes with nonzero eigenvalue indeed have an associated zero linear perturbation in angular momentum. It is also verified that in the case where $N=0$ (so that the steady state is the monopolar shielded Rankine vortex) the spectrum found analytically in Sec. III A is retrieved.

A note on a limitation of the numerical method. The method uses a Taylor expansion (48) of $F(z, t)$ and as $a$ $\rightarrow a_{\text {crit }}^{(N)}$ it must be expected that it will become increasingly inaccurate for fixed values of $\mathcal{N}$ and $\mathcal{M}$ as a result of attempting to evaluate an expansion close to its radius of convergence. Indeed, for $a$-values sufficiently far from $a_{\text {crit }}^{(N)}$ it is found that independently increasing $\mathcal{N}$ and $\mathcal{M}$ does not affect the results of the eigenvalue calculation; however if $a$ is too close to $a_{\text {crit }}^{(N)}$ the spectrum is susceptible to significant changes as the order of the spectral method is increased. Thus, using the above method, the linear stability of solutions with values of $a$ too close to $a_{\text {crit }}^{(N)}$ cannot be reliably computed.

\section{Results}

Results are presented for $\omega=1$; growth rates for other values of $\omega$ are obtained by multiplication. For each $N \geqslant 2$, the maximum linear growth rate (i.e., the magnitude of the real part of the eigenvalue with the largest positive real part) is calculated for different values of $a$ within the range of existence of the solutions. From (16), it is seen that the values of $a_{\text {crit }}^{(N)}$ are all around 1.25 for $N=2, \ldots, 5$. Using $\mathcal{N}$ $=64$, it is found that the numerical method just described can adequately resolve the linear stability spectrum for $a \geqslant 1.35$ (although for $N=2, a=1.4$ is the smallest value that can be used reliably).

It is found that the tripolar solutions with $N=2$ are linearly unstable for all choices of $a$. The maximum linear growth rate in this case is shown in Fig. 9. The case $N=3$ is distinguished and the solutions are found to be neutrally stable for all $a$ values that can be calculated reliably using the method employed. While no eigenvalues with nonzero real part are found, seven zero eigenvalues are found. This is true for all other values of $N$. One corresponds to a neighboring steady state with different area, another two correspond to modes in which the vortex centroid is displaced. The remaining four zero eigenvalues appear to correspond to nontrivial neutral states indicating the possibility of neighboring steady solutions, but this is not investigated further here. For present purposes, the important fact is that no eigenvalues with nonzero real part are found.

For the cases $N=4,5$, and 6 which were investigated in detail, it is found that for large $a$ the solutions are linearly unstable. However, as $a$ falls below some critical value (denoted $\left.a_{\text {stab }}^{(N)}\right)$ the configurations become neutrally stable, with any real eigenvalues passing through the origin and becoming pure imaginary. Calculations using $\mathcal{N}=64$ produce the values $a_{\text {stab }}^{(4)}=1.40$ (correct to 2 decimal places), $a_{\text {stab }}^{(5)}=1.41$ and $a_{\mathrm{stab}}^{(4)}=1.37$. Graphs of the maximum linear growth rates 


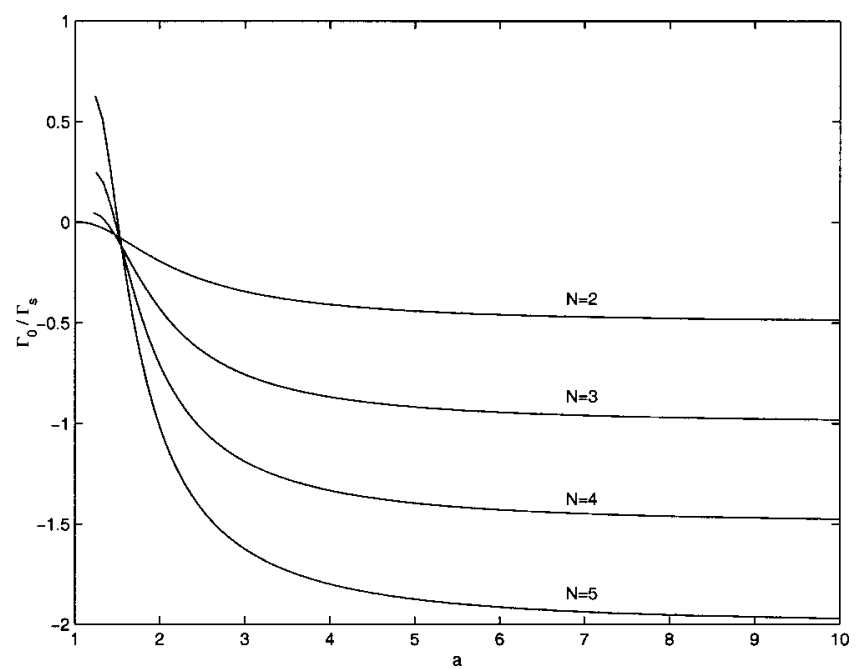

FIG. 10. Graph of $\Gamma_{0} / \Gamma_{s}$ for $N=2,3,4$, and 5. As $a \rightarrow \infty$, ratio approaches asymptotic value given by (58).

are shown in Fig. 9. It is conjectured that values of $a_{\text {stab }}^{(N)}$ at which there is an exchange of stability exist for all higher values of $N$. This was checked explicitly for $N$ up to 8 .

It is also observed from inspection of the corresponding eigenmodes for large $a$ and $M=2$ that the most unstable eigenmodes are associated with displacement of the central line vortex while for $M=4$ and $M=5$ the most unstable modes are associated with displacement of the satellite line vortices with no motion of the central line vortex.

The work of Morikawa and Swenson ${ }^{19}$ involving line vortices is consistent with many of these results, especially in the parameter range $a \rightarrow \infty$. For large $a$, the vortex patch becomes large compared to the separation of the line vortices and it becomes near-circular in shape. The role of the patch can then be interpreted as providing an overall bulk rotation such as to cancel the rotation that the line vortex configuration would otherwise have if placed in a field of irrotational fluid. In this case, it is reasonable to expect that the stability properties of the localized line vortex configuration might be inherited from the results of Morikawa and Swenson. ${ }^{19}$ An asymptotic analysis of (2) as $a \rightarrow \infty$ reveals that

$$
b \sim \frac{2 N a^{N}}{(N-1)} \quad \text { as } a \rightarrow \infty .
$$

Using (1) and (8) it follows that

$$
\frac{\Gamma_{0}}{\Gamma_{s}} \sim-\frac{(N-1)}{2} \text { as } a \rightarrow \infty .
$$

This fact facilitates comparison with Table I on p. 1063 of Morikawa and Swenson ${ }^{19}$ which provides the stability region for a configuration of $N$ equal satellite line vortices surrounding a central line vortex as a function of the ratio of the central-to-satellite circulations. Comparison with Table I of Ref. 19 shows that the asymptotic ratio $-(N-1) / 2$ only falls within the stability range in the case $N=3$. Figure 10 shows that as $a$ decreases, $\Gamma_{0} / \Gamma_{s}$ increases for each $N$. When $N=3, \Gamma_{0} / \Gamma_{s}$ falls within the stability range of Ref. 19 for all values of $a$. For $N=2, \Gamma_{0} / \Gamma_{s}$ never falls inside the stability region of Ref. 19 for any value of $a$ while, in the other two cases $N=4$ and 5 shown in Fig. 10, this ratio falls within the stability range of Ref. 19 for sufficiently small $a$. Indeed, $\gamma_{0}=-0.5$ is the critical value for stability found by Morikawa and Swenson ${ }^{19}$ for $N=4$ and 5. Here, when $N$ $=4, \Gamma_{0} / \Gamma_{s}=-0.5$ when $a=1.808$, while the relevant value of $N=5$ is $a=1.697$. It should not be expected that these values will correspond to $a_{\text {stab }}^{(4)}$ and $a_{\text {stab }}^{(5)}$ because, for these small values of $a$, the nontrivial shape of the ambient vortex patch must be assumed to be having some effect on the equilibrium other than simply cancelling out the rotation that the line vortex configuration would otherwise have if the patch is absent. Nevertheless, the above linear stability results are qualitatively consistent with Morikawa and Swenson. ${ }^{19}$

The above results are further consistent with Morikawa and Swenson ${ }^{19}$ in that, for $N=2$, they also find that the instability is associated with perturbations of the central line vortex while, for $N=4$, it is the satellite vortices that are unstable.

\section{NONLINEAR EVOLUTION}

The purpose of this section is to examine to what extent the results of the linear stability analysis manifest themselves in the nonlinear evolution of the perturbed equilibria of Ref. 1. It is not our aim to present an exhaustive study of the nonlinear stability of the solutions.

A fortuitous property of the class of exact solutions found in Crowdy ${ }^{1}$ is that they are a hybrid of two of the most well-studied mathematical idealizations of two-dimensional vortex dynamics, i.e., line vortices and uniform vortex patches. This important fact implies that the nonlinear evolution of this class of solutions can be studied using contour dynamics methods as originally expounded by Deem and Zabusky. ${ }^{12}$ An adapted version of the contour surgery code as developed by Dritschel ${ }^{20}$ is now used to examine the typical nonlinear evolution of the multipolar solutions of Ref. 1 .

The contour surgery code of Dritschel ${ }^{20}$ is modified by adding $2 N+2$ nonlinear ordinary differential equations governing the $x$ and $y$ positions of the $N+1$ line vortices superposed on the vortex patch. By the Helmholtz vortex theorems ${ }^{18}$ it is known that line vortices move with the nonself-induced local velocity. Several runs of the modified code consisting purely of an $\mathrm{N}$-polygonal array of co-rotating line vortices is made for various values of $N$. The angular velocity $\Omega$ of steady rotation is well known to be given as

$$
\Omega=\frac{\bar{\Gamma}(N-1)}{4 \pi r_{0}^{2}},
$$

where $r_{0}$ is the radial distance of each line vortex from the origin, and $\bar{\Gamma}$ is the circulation of each vortex. ${ }^{18}$ This provides a check that the supplementary equations describing the line vortex motion have been correctly added to the contour dynamics algorithm.

In the following calculations we choose $\omega=1$. In the calculations which follow, time is scaled with $2 \pi$. 

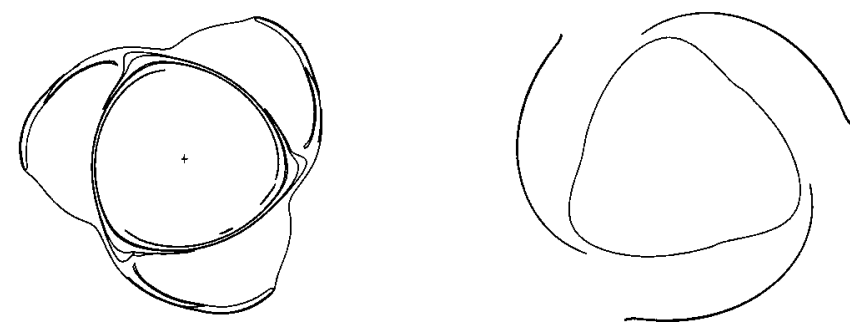

FIG. 11. Vortical configuration at $t=3$ of shielded Rankine vortex (left diagram) and regular Rankine vortex (right diagram) perturbed by azimuthal mode- 3 perturbation of the form (60) with amplitude $A=0.4$. In contrast to the regular Rankine vortex, the shielded vortex forms a distinctly quadrupolar configuration.

\section{A. Shielded Rankine vortex}

While it was shown in Sec. III A that the shielded Rankine vortex solution is neutrally stable, this implies nothing about its nonlinear stability. In this section, we show the results of imposing the following azimuthal-mode $m$ perturbation to the circular boundary $r=1$ (where $r$ denotes the usual radial polar coordinate) of the shielded Rankine vortex

$$
r=1+A \cos m \theta,
$$

where $\theta$ is the polar angular variable and $A$ represents a measure of the size of the perturbation. In linear theory, $A$ is taken to be small; however, we do not impose this restriction. We also show the results of perturbing a regular Rankine vortex to exactly the same perturbation.

The purpose is to examine whether a shielded Rankine vortex, if perturbed strongly enough, can nonlinearly destabilize into a multipolar vortex structure. Carton ${ }^{4}$ has shown that provided the initial perturbation is strong enough, the two-contour Rankine vortex can destabilize into higher-order multipolar structures although they tend not to be long lived. Morel and Carton ${ }^{7}$ show that a three-contour Rankine vortex destabilizes into higher-order multipolar structures. As mentioned in Ref. 1, the shielded Rankine vortex is a limit of the (shielded) two-contour Rankine vortex in which the enclosed central patch of vorticity is taken to a line vortex limit. It is therefore feasible that a suitably perturbed shielded Rankine vortex might exhibit similar qualitative behavior to the unstable monopoles considered in Ref. 7.

Figures 11 and 12 answer this question in the affirmative. Figure 11 shows the vortical configuration, at time $t$ $=3$, of a shielded Rankine vortex with an initial mode- 3 perturbation of the kind (60) of magnitude $A=0.4$. This represents a finite-amplitude perturbation and it is clear that the vortex boundary folds in on itself in such a way as to form a very distinct quadrupolar structure. A threefold rotational
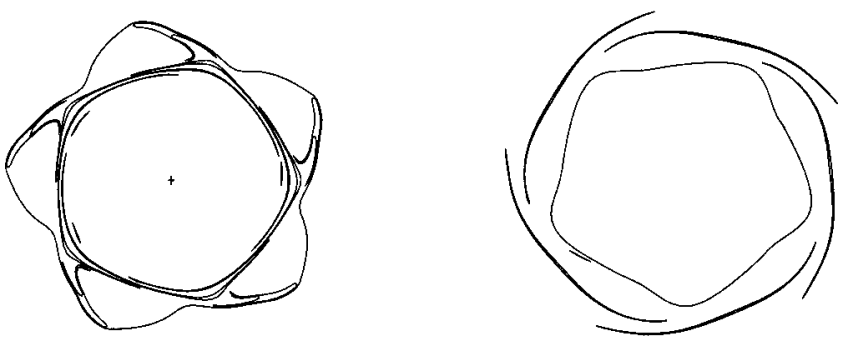

FIG. 12. Vortical configuration at $t=3$ of shielded Rankine vortex (left diagram) and regular Rankine vortex (right diagram) perturbed by azimuthal mode- 5 perturbation of the form (60) with amplitude $A=0.3$. In contrast to the regular Rankine vortex, the shielded vortex forms a distinctly sextapolar configuration.

symmetry is generally preserved and each satellite vortex region has roughly the same circulation which is opposite to the overall circulation of the central region. Also shown (rightmost) in Fig. 11 is the state of a regular Rankine vortex at the same time in the evolution, having been exposed to exactly the same perturbation. While the residues of thin filaments that have been excised from the patch during the evolution are clearly seen, there is no well-defined multipolar formation in this case. Figure 12 shows the response after $t$ $=3$ of a mode -5 perturbation of slightly smaller amplitude $A=0.3$ and it is clear that a well-defined sextapolar structure has developed. In contrast, the state at $t=3$ of a similarly perturbed regular Rankine vortex (also shown) does not exhibit the same distinctive multipolar formation. These preliminary nonlinear calculations highlight the crucial role played by the presence of the central line vortex in the formation of multipolar structures.

\section{B. General equilibria of Ref. 1}

First, several runs of the contour surgery code are made in which the initial vortical configurations are given with no perturbation added. The contour surgery threshold parameter is set at 0.1 so that there are typically between $100-150$ points on each contour. More distorted shapes are described using more points. The time step is also chosen carefully in order to avoid stiffness problems when $a$ is large so that the line vortices are close together.

Figures 13-15 show the responses, by time $t=5$, of unperturbed tripolar, quadrupolar and pentapolar vortices. In all cases, the initial states are seen to remain stationary under evolution of the contour dynamics equations for a certain period of time. Eventually, small numerical errors seed the growth of any linearly unstable modes. The tripole in Fig. 13 is seen to asymmetrically decompose into a monopolar and dipolar structure. The initial stages of the instability are char-
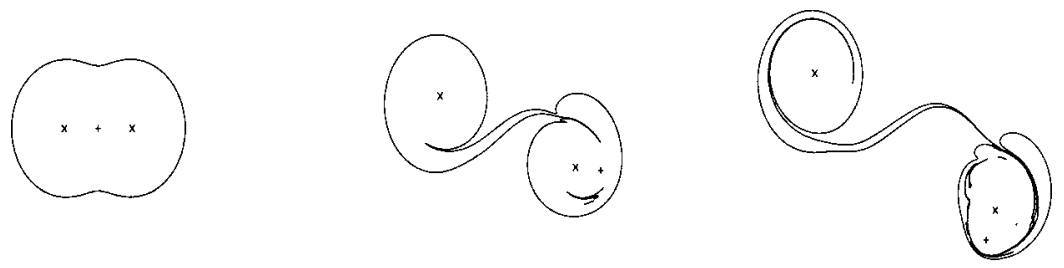

FIG. 13. Evolution of an unperturbed $N=2, a=2$ vortex at times $t=0,3.25$, and 5. The tripole splits into a monopole and a dipole by growth in an instability in which the central line vortex becomes displaced. 

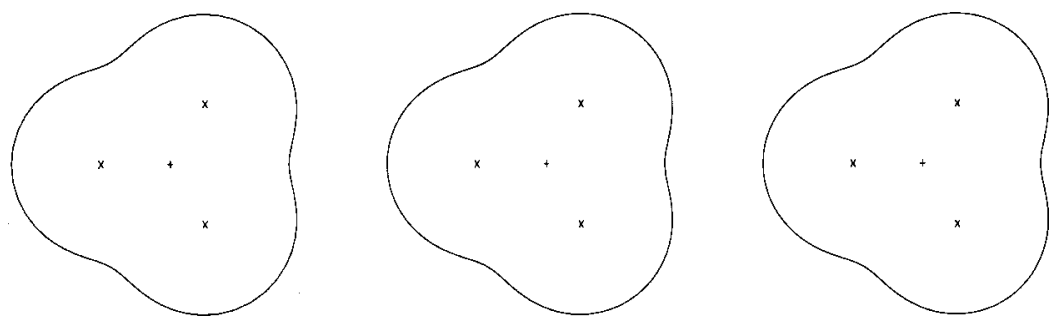

FIG. 14. Evolution of an unperturbed $N=3, a=2$ vortex at times $t=0,3.25$, and 5 . No detectable change in the vortex is observed (even after much longer times).

acterized by the central line vortex moving off its equilibrium position. This is consistent with the linear stability analysis: the displacements of the line vortices associated with the most unstable eigenmode of the linear stability analysis are also found to be dominated by displacements of the central line vortex with much smaller displacements of the satellites. A similar phenomenon of asymmetric decomposition into monopole and dipole is observed by Morel and Carton $^{7}$ for a particular tripole although most configurations investigated by these authors prove to be stable. For the class of tripoles found in Ref. 1, none are linearly stable.

On the other hand, Fig. 14 highlights the fact that a quadrupole remains stationary and unperturbed for extended periods. This is consistent with the linear stability analysis which reveals that there are no unstable linear modes in this case. This calculation also provides a reassuring check that the contour surgery code has been correctly adapted to take account of the superposed line vortices.

Figure 15 shows the decomposition into a dipole and three monopoles of an initially unperturbed pentapolar vortex with parameters $N=4$ and $a=2.0$. This configuration has a maximum linear growth rate of $\sigma \approx 0.75$ and, distinct from the case of the tripole where the most unstable eigenmode is predominantly associated with displacements of the central line vortex, here the most unstable eigenmode has zero perturbation of the central vortex and only displacements associated with the satellite line vortices. This is consistent with the nonlinear evolution where the satellites are seen to destabilize first with the central vortex position unchanged (see the middle diagram of Fig. 15). It is worth pointing out that, for certain values of $a$, the satellite line vortices were sometimes seen to engage in a curious "dance" around the central line vortex, with the patch remaining mostly stationary. Morikawa and Swenson ${ }^{19}$ observed similar phenomena in the case of even numbers of satellite line vortices and provided the perturbation size was small enough. They referred to them as "nonlinear periodic oscillations." This line vortex phenomenon may be related to a relaxation oscillation asso- ciated with a heteroclinic orbit in the dynamics of a 5-line vortex system as recently studied by Nakaki. ${ }^{21}$

The nonlinear evolution of perturbed equilibria is now examined. The class of perturbations which have been considered in most detail are those in which all satellites are symmetrically displaced inwards or outwards by a certain amount. To show the effect of this perturbation on linearly stable configurations, Fig. 16 shows the nonlinear response of an $a=1.37$ quadrupolar vortex to a perturbation in which all satellite line vortices are initially displaced to a radial distance 1.1 from the central line vortex. Minor rearrangement of the patch vorticity takes place but the configuration is structurally robust and, after a short period of adjustment, the overall structure rotates in an anticlockwise direction. If the satellites are moved inwards by 0.1 , there is a more dramatic rearrangement of the patch vorticity but again the structure retains its overall quadrupolar form and rotates in a clockwise direction. This behavior of the perturbed quadrupoles with the associated reorganization of the vorticity (which includes some filamentation events and surgery and therefore small losses in total circulation) is highly suggestive of the configuration adjusting itself to relax into a neighboring (rotating) equilibrium. (See Fig. 17.)

We now explore the nonlinear evolution of pentapolar structures on either side of the linear stability boundary. Figure 18 shows the evolution of an $a=1.65$ pentapolar vortex when all satellites are moved outwards by 0.1 . Figure 19 shows the evolution of a pentapolar structure with $a=1.4$ under the same perturbation. The former configuration has a linear growth rate of approximately 0.35 while the latter sits on the linear stability boundary. In common with the linearly stable quadrupolar vortices, it is found that the $a=1.4$ pentapolar vortex undergoes a mild rearrangement of the patch vorticity and rotates (anticlockwise) without any significant change of structural form right up to $t=10$. In contrast, by $t=7.5$, the $a=1.65$ vortex structure has disintegrated completely.

The results of a large number of experimental runs sug-
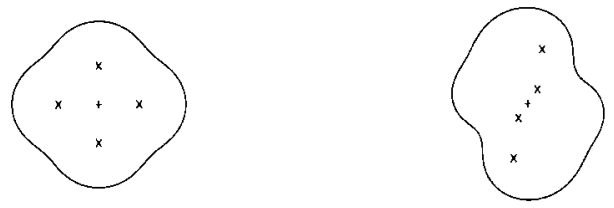

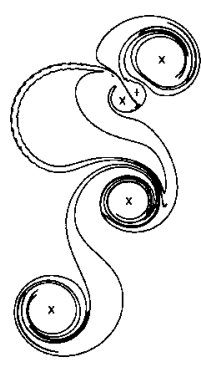

FIG. 15. Evolution of an unperturbed $N=4, a=2$ vortex at times $t=0,3.25$, and 5. The vortex is seen to decompose into three monopoles and a dipole by growth in the displacements of the satellite line vortices. 

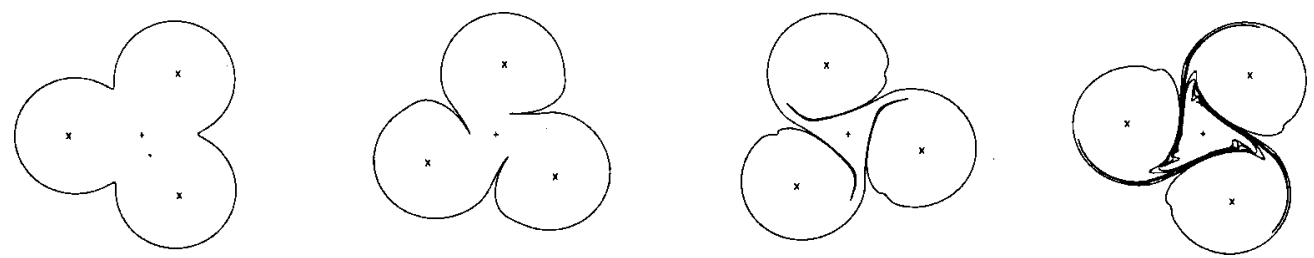

FIG. 16. Nonlinear response of a quadrupolar vortex $(a=1.37)$ to a perturbation in which all satellite line vortices (each of strength $\left.\Gamma_{s}=2.563\right)$ are moved outwards by 0.1 . The strength of the central line vortex is negligible. A slight rearrangement of the patch vorticity occurs and the structure rotates anticlockwise but retains its overall quadrupolar form. Times shown are $t=0,1,2$, and 5 .
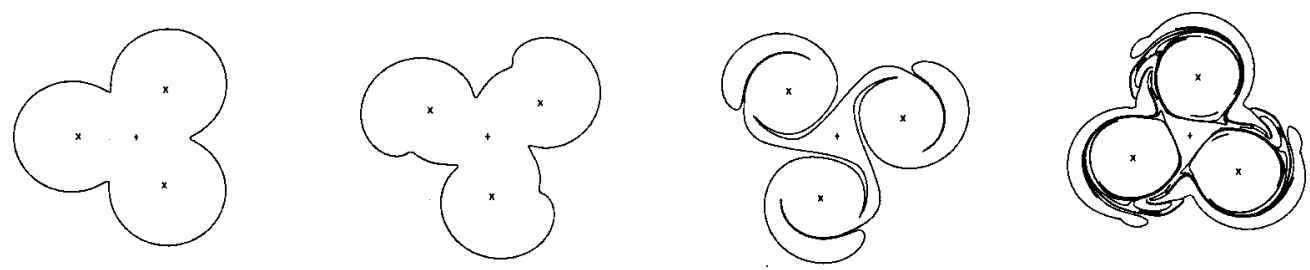

FIG. 17. Nonlinear response of a quadrupolar vortex $(a=1.37)$ to a perturbation in which all satellite line vortices (each of strength $\left.\Gamma_{s}=2.563\right)$ are moved inwards by 0.1 . The strength of the central line vortex is negligible. There is a more dramatic rearrangement of the patch vorticity than in Fig. 16 but the structure retains its overall quadrupolar form and rotates steadily in a clockwise direction. Times shown are $t=0,1,2$, and 5 .
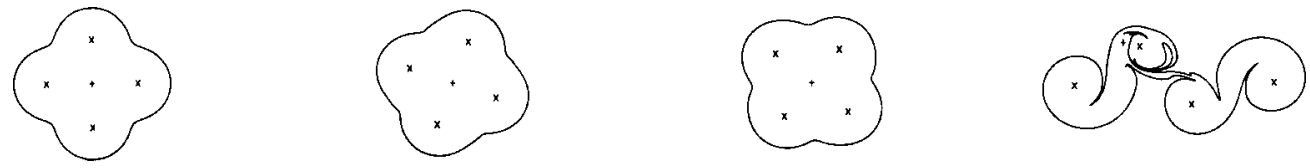

FIG. 18. Nonlinear response of a pentapolar vortex $(a=1.65)$ to a perturbation in which all satellite line vortices are moved outwards by 0.1 . This structure is linearly unstable with a linear growth rate of approximately 0.1 . The structure rotates steadily for a while but by $t=7.5$ (roughly the $e$-fold time according to linear theory) it has eventually disintegrated. Times shown are $t=0,2.5,5$, and 7.5.
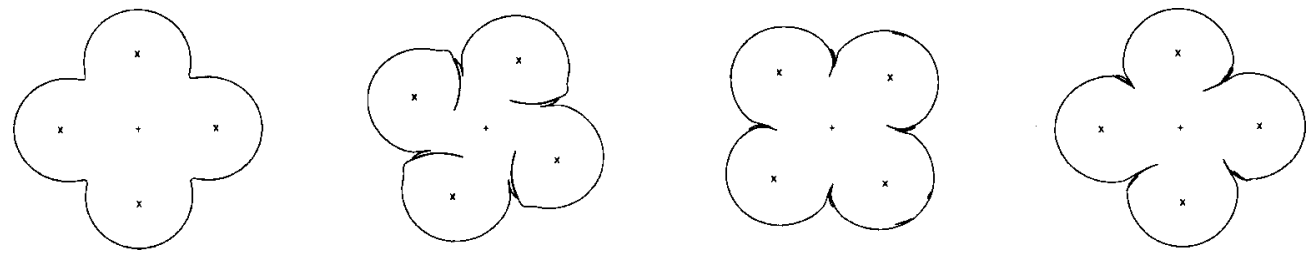

FIG. 19. Nonlinear response of a pentapolar vortex $(a=1.4)$ to a perturbation in which all satellite line vortices are moved outwards by 0.1 . This structure sits on the linear stability boundary. The structure is slowly rotating anticlockwise and retains its pentapolar form. Times shown are $t=0,2.5,5$, and 10 .
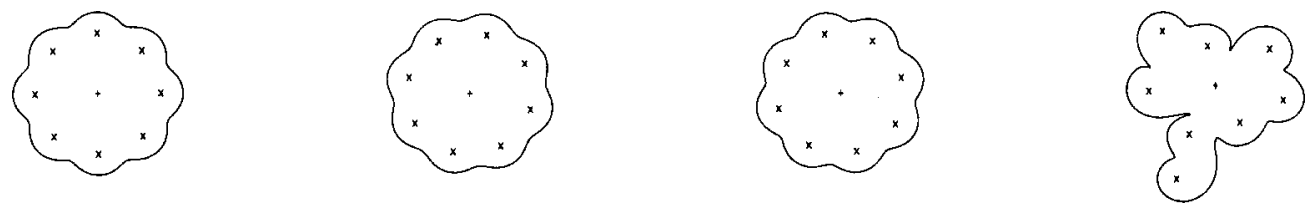

FIG. 20. Nonlinear response of an 8-polar vortex with $a=1.4$ to a perturbation in which all satellite line vortices are moved outwards by 0.1 . This configuration has a maximum linear growth rate of 0.31 . Times shown are $t=0,2.5,5$, and 7.5 . The configuration rotates steadily for a while, but by $t$ $=7.5$ (roughly the $e$-fold time according to linear theory) the structure has disintegrated.
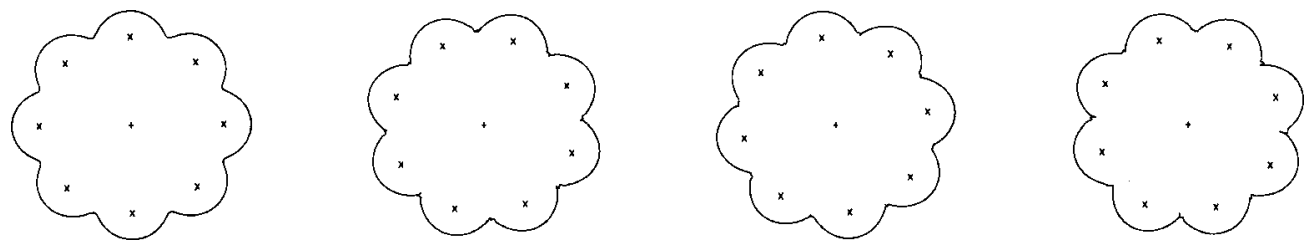

FIG. 21. Nonlinear response of an 8-polar vortex with $a=1.3$ to a perturbation in which all satellite line vortices are moved outwards by 0.1 . Times shown are $t=0,2.5,5$, and 10 . This structure is neutrally stable according to linear theory. The configuration rotates steadily and maintains its structural form under evolution. Note that small ripples in the boundary occur at the points of highest curvature. 
gest that the linear growth rates provide a good guide to what happens to the vortical structures under perturbations of this kind. If an unperturbed vortex is linearly unstable then it is generally found to have disintegrated by roughly its $e$-fold time according to linear stability theory. For example, vortices with linear growth rates of order $10^{-1}$ are typically found to have disintegrated by around $t=10$.

On the other hand, linearly stable configurations are found to simply rotate steadily for extended periods of time. An additional illustration of this is provided in Figs. 20 and 21 which show configurations, with $a=1.4$ and $a=1.3$, respectively, for $N=8$. Again, the equilibria are perturbed by moving the satellites outwards by 0.1. As in the pentapolar case, the $a=1.4$ case (which, it is found, has a maximum linear growth rate of 0.31 ) has disintegrated by $t=7.5$, while the $a=1.3$ configurations (which are neutrally stable according to linear theory) rotates steadily up to the termination of the calculations. These results suggest that this perturbation constitutes a linear one. In general, it is reasonable to expect that what constitutes a linear perturbation will depend on the equilibrium being perturbed. Nevertheless, these limited nonlinear calculations reveal that linear stability theory can provide a useful guide to the nonlinear fate of these vortex structures. Moderate nonlinear perturbation seems generally to result in one of two long-term consequences: a steadily rotating structure or complete structural disintegration. The linear stability boundaries seem to provide a good working guide to which of these two eventualities occurs in any particular case.

\section{DISCUSSION}

The stability properties of the exact solutions in Ref. 1 have been found to be consistent with results on the stability of multipolar vortices obtained by previous investigators except in the case of the tripolar solutions within this class. It is known that tripoles can be stable structures while those found in Ref. 1 appear to be linearly unstable in all admissible configurations. It has been found that only the quadrupoles are neutrally stable in all configurations-a result consistent with general observations on the robustness of quadrupoles made by previous investigators. It seems to be generally accepted in the literature that tripoles and quadrupoles are the only possible stable structures. In contrast, the above results illustrate that there exist zero-total-circulation multipolar structures of order $N \geqslant 4$ (i.e., pentapoles and higher) which are both neutrally stable and nonlinearly robust, although this is only true provided $a$ is sufficiently small. Physically, this corresponds to the satellites being far enough away from the center of the structure and the ambient patch sufficiently deformed. It is interesting that, in their investigations of a rather different class of pentapolar structures evolving in a contour surgery simulation, Morel and Carton also report (in Table 2 of Ref. 7) an example of an apparently stable pentapole.

As for the tripolar solutions, we conjecture that the combined constraints that the total structure has zero circulation and be nonrotating force the respective strengths of the central and satellite line vortices to be such that linear stability of the configuration is not possible. Perhaps generalized equilibria involving line vortices superposed on a patch which are allowed to rotate steadily will produce a class of stable tripolar structures. It is unlikely, however, that this generalized rotating class will be describable in exact mathematical form (although, it is worth pointing out that a class of exact rotating equilibria have recently been found ${ }^{14}$ using an analysis similar to that used in Ref. 1). It must be remembered that the solutions of Ref. 1 are a mathematically exact subclass (and hence, a rather special class) of the general class of multipolar vortices. A priori, there is no reason to suppose that this special class will share any of the stability properties of the general class even though the exact equilibria exhibit many of the defining qualitative features of the more general class (see the discussion in Ref. 1). However, as this paper has shown, the tripolar solutions appear to be the only ones whose stability properties are atypical of general tripolar vortices.

Owing to the choice of method used, it has not been possible to study the linear stability of the limiting configurations discussed in Sec. I, nor indeed, configurations that are too close to limiting. However, several calculations for different $N$ were performed for $a$ values close to $a_{\text {crit }}^{(N)}$ and with the order of the spectral method taken to be much larger than the $\mathcal{N}=64$ used to obtain the results reported here. In all such calculations, as the order of the spectral method is increased, the real parts of the spectrum tended to disappear thereby suggesting that the states very close to limiting are in fact neutrally stable. However, we make no definite statement on this matter until a method capable of more accurately computing these linear stability spectra is implemented.

\section{ACKNOWLEDGMENTS}

D.C. wishes to thank Professor H. Aref and Professor D. Dritschel for useful discussions during a workshop entitled "Geometry and Topology of Fluid Flows" held at the Newton Institute in Cambridge in December 2000. D.C. also thanks Professor Dritschel for explaining his contour surgery code. This research is partially supported by a grant from the Nuffield Foundation.

${ }^{1}$ D. G. Crowdy, “A class of exact multipolar vortices," Phys. Fluids 11, 2556 (1999).

${ }^{2}$ G. R. Flierl, "On the instability of geostropic vortices," J. Fluid Mech. 197, 349 (1988).

${ }^{3}$ L. M. Polvani and X. Carton, "The tripole: a new coherent structure of incompressible two-dimensional flows," Geophys. Astrophys. Fluid Dyn. 51, 87 (1990).

${ }^{4}$ X. J. Carton, "On the merger of shielded vortices," Europhys. Lett. 18, 697 (1992).

${ }^{5}$ S-J. Lin, "Contour dynamics of tornado-like vortices," J. Atmos. Sci. 49, 1745 (1992).

${ }^{6}$ G. F. Carnevale and R. C. Kloosterziel, "Emergence and evolution of triangular vortices," J. Fluid Mech. 259, 305 (1994).

${ }^{7}$ Y. G. Morel and X. J. Carton, "Multipolar vortices in two-dimensional incompressible flows," J. Fluid Mech. 267, 23 (1994).

${ }^{8}$ K. Fine, A. Cass, W. Flynn, and C. F. Driscoll, "Relaxation of 2D turbulence to vortex crystals," Phys. Rev. Lett. 75, 3277 (1995).

${ }^{9}$ D. Durkin and J. Fajans, "Experiments on two-dimensional vortex patterns," Phys. Fluids 12, 289 (2000). 
${ }^{10}$ D. Durkin and J. Fajans, "Experimental dynamics of a vortex within a vortex," Phys. Rev. Lett. 85, 4052 (2000).

${ }^{11}$ D. Z. Jin and D. H. E. Dubin, "Point vortex dynamics within a background vorticity patch," Phys. Fluids 13, 677 (2001).

${ }^{12}$ G. S. Deem and N. J. Zabusky, "Vortex waves: stationary V-states, interactions, recurrence and breaking," Phys. Rev. Lett. 40, 859 (1978).

${ }^{13}$ E. A. Overman, "Steady-state solutions of the Euler equations in two dimensions II: local analysis of limiting V-states," SIAM (Soc. Ind. Appl. Math.) J. Appl. Math. 46, 765 (1986).

${ }^{14}$ D. G. Crowdy, "Exact solutions for rotating vortex arrays with finite-area cores," J. Fluid Mech. (in press).

${ }^{15}$ D. G. Crowdy, "The construction of multipolar equilibria of the 2D Euler equation," Phys. Fluids 14, 257 (2002).

${ }^{16}$ D. G. Crowdy, "Multipolar vortices and algebraic curves," Proc. R. Soc. London, Ser. A 457, 2337 (2001).
${ }^{17}$ D. I. Meiron, P. G. Saffman, and J. C. Schatzman, "The linear two dimensional stability of inviscid vortex streets of finite-cored vortices," J. Fluid Mech. 147, 187 (1984).

${ }^{18}$ P. G. Saffman, Vortex Dynamics (Cambridge University Press, Cambridge, UK, 1992).

${ }^{19}$ G. K. Morikawa and E. V. Swenson, "Interacting motion of rectilinear geostrophic vortices,” Phys. Fluids 14, 1058 (1971).

${ }^{20}$ D. Dritschel, "Contour surgery: a topological reconnection scheme for extended interactions using contour dynamics," J. Comput. Phys. 77, 511 (1988).

${ }^{21}$ T. Nakaki, "Behavior of point vortices in a plane and existence of heteroclinic orbits," Dynamics of Continuous, Discrete and Impulsive Systems, 5, 159 (1999). 